\title{
Proteomic analysis of enterotoxigenic Escherichia coli (ETEC) in neutral and alkaline conditions
}

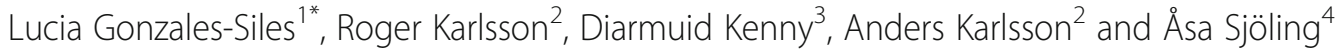

\begin{abstract}
Background: Enterotoxigenic Escherichia coli (ETEC) is a major cause of diarrhea in children and travelers to endemic areas. Secretion of the heat labile $A B_{5}$ toxin $(L T)$ is induced by alkaline conditions. In this study, we determined the surface proteome of ETEC exposed to alkaline conditions ( $\mathrm{pH}$ 9) as compared to neutral conditions (pH 7) using a LPI Hexalane FlowCell combined with quantitative proteomics. Relative quantitation with isobaric labeling (TMT) was used to compare peptide abundance and their corresponding proteins in multiple samples at MS/MS level. For protein identification and quantification samples were analyzed using either a 1D-LCMS or a 2D-LCMS approach.

Results: Strong up-regulation of the ATP synthase operon encoding F1Fo ATP synthase and down-regulation of proton pumping proteins NuoF, NuoG, Ndh and WrbA were detected among proteins involved in regulating the proton and electron transport under alkaline conditions. Reduced expression of proteins involved in osmotic stress was found at alkaline conditions while the Sec-dependent transport over the inner membrane and outer membrane protein proteins such as OmpA and the $\beta$-Barrel Assembly Machinery (BAM) complex were upregulated.
\end{abstract}

Conclusions: ETEC exposed to alkaline environments express a specific proteome profile characterized by upregulation of membrane proteins and secretion of $L T$ toxin. Alkaline microenvironments have been reported close to the intestinal epithelium and the alkaline proteome may hence represent a better view of ETEC during infection.

Keywords: ETEC, pH regulation, Proteomics, Alkaline, ATPase, OmpA, BAM

\section{Background}

Enterotoxigenic Escherichia coli (ETEC) remains to be one of the major causes of childhood diarrhea and is a global health problem [1]. ETEC cause disease by adhering to the epithelium of the small intestine by means of different colonization factors [2]. The two major ETEC toxins, heat labile toxin (LT) and heat stable toxin (ST), binds to enteric receptors on the epithelium and ultimately cause de-regulation of the chloride channel CFTR, which leads to increased secretion of chloride ions, bicarbonate and electrolytes [3]. LT is an $\mathrm{AB}_{5}$ toxin encoded by the elt $A$ and eltB genes in one operon. The LTA and LTB peptides are secreted through sec dependent mechanisms

\footnotetext{
*Correspondence: lucia.gonzales@gu.se

'Department of Infectious Disease, Institute of Biomedicine, Sahlgrenska

Academy, University of Gothenburg, SE-41346 Gothenburg, Sweden

Full list of author information is available at the end of the article
}

to the periplasm and assembled by DsbA [4]. Secretion through the outer membrane occurs via the Type II secretion system (T2SS) [5]. Secretion of LT has been reported to vary between ETEC isolates, ranging from being completely retained in the periplasm [6], to secretion of up to $50 \%$ of the produced LT holotoxin in LB media [7-9]. The ST toxin is also transported in a Sec-dependent manner through the inner membrane but is released through TolC [10]. The small ST peptide is cleaved and folded in the process and the mature peptide is secreted to the outer environment.

ETEC encounter different environments in the human gastrointestinal tract before reaching optimal conditions for infection in the small intestine and environmental cues, such as bile, oxygen and $\mathrm{pH}$ affect secretion of toxins and virulence of ETEC [7, 11, 12]. Passage through the stomach exposes infecting pathogens to 
acidic conditions, while entry into the duodenum is characterized by a rise of $\mathrm{pH}$ due to release of bile and bicarbonate [13, 14]. Further down in the anaerobic gut the $\mathrm{pH}$ is expected to drop to acidic levels again but close to the small intestinal epithelium alkaline conditions can occur due to release of bicarbonate. Alkaline surface microclimates in the small intestine have been described previously [15]. ETEC toxins ST and LT both enhance secretion of bicarbonate through activation of the CFTR ion channel, which might create an extremely alkaline microenvironment close to the infecting bacteria. Interestingly, similar to the highly homologous cholera toxin $(\mathrm{CT})$ the assembly of LT seems to be dependent on an alkaline environment $[7,16,17]$. We have previously shown that secretion of LT toxin is favored under alkaline conditions and inhibited under acidic conditions [7]. Hence our results support the hypothesis that ETEC toxin secretion is induced at alkaline conditions at the site of infection. In this study we analyzed the proteome of ETEC exposed to alkaline conditions ( $\mathrm{pH}$ 9) as compared to neutral conditions ( $\mathrm{pH} 7$ ) in order to further determine the effect of highly alkaline conditions on ETEC.

\section{Methods}

\section{Overview of methodology}

Clinical isolate ETEC E2863 was cultured in either $\mathrm{pH} 7$ or $\mathrm{pH} 9 \mathrm{LBK}$ media at three separate occasions to produce three biological replicates. For each biological replicate, we include three technical replicates. The bacteria culture for each $\mathrm{pH}$ condition was immobilized and digested in three separate LPI Hexalane channels generating three separate peptide samples (Fig. 1). Peptide samples generated for both $\mathrm{pH}$ conditions were labelled with the TMT (6-plex) kit and combined into one set. The set was then split into two aliquots for analysis with either 1D-LC or 2D-LC fractionation followed by MS analysis (Fig. 1). Following MS analysis and database

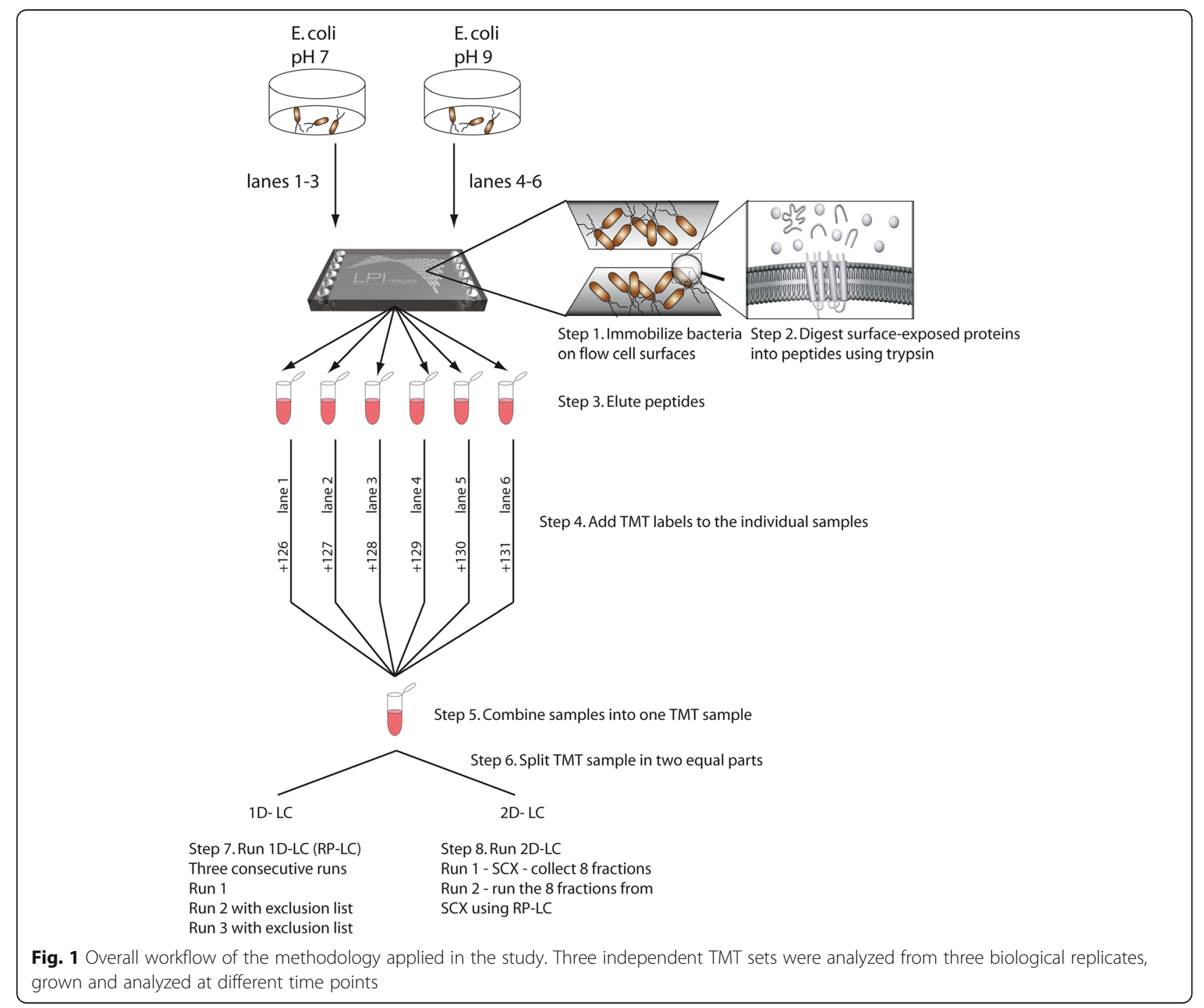


matching relative quantification was performed. Proteins displaying more than $20 \%$ variation between the three samples from the individual LPI channels at each condition were removed. This was done by calculation the ratio of the separate TMT-labels in a group, and the average of the combined channels e.g. 126/(average $126+$ $127+128$ ). Proteins with rations between 0.8 and 1.2 were included in the protein list. For comparison of the two conditions, fold changes were calculated and a statistical analysis Welch's $t$-test was used for multiple comparisons. Only proteins passing the statistical filter $(p<0.05)$ were accepted. Additionally, all three biological replicates, were statistically evaluated as described above resulting in three separate lists of quantified proteins considering a fold change of at least 1.5 as a threshold for considering relevant up or down regulation. Finally, the proteins accepted for the biological interpretation were quantified in at least two of the three TMT-sets and biological replicates.

\section{Culture conditions}

The ETEC clinical isolate E2863 was used in the study. E2863 was grown in LBK media (10 g Tryptone, 5 g yeast extract, $6.4 \mathrm{~g} \mathrm{KCl}$ ) buffered to $\mathrm{pH} 7$ using piperazine- $\mathrm{N}$, N9-bis-(2-ethanesulfonic acid) (PIPES) or pH 9 using 3[(1,1-dimethyl-2-hydroxyethyl)amino]-2-hydroxypropanesulfonic acid (AMPSO) at $100 \mathrm{mM}$. Media were adjusted for $\mathrm{pH}$ with $\mathrm{KOH}$, to avoid high concentrations of sodium ions, which inhibit growth at high $\mathrm{pH}$. These buffers help cultures to maintain a constant $\mathrm{pH}$ throughout growth. All cultures were grown for $3 \mathrm{~h}$ since the highest secretion levels of LT toxin has been reported to occur at this time [7], $\mathrm{pH} 7$ cultures reached an $\mathrm{OD}_{600}$ of 1.2 whereas $\mathrm{pH} 9$ cultures reached an $\mathrm{OD}_{600}$ of 0.4 .

\section{Trypsin digestion of bacteria in LPI HexaLane FlowCell and TMT (tandem mass tags) labeling}

The bacterial biomass was washed with PBS three consecutive times by centrifugation of the samples for $5 \mathrm{~min}$ at $10.000 \mathrm{rpm}$, followed by discarding the supernatant and then resuspending the pellet in $1 \mathrm{ml}$ PBS. The washed bacterial suspension was injected into the LPI Hexalane FlowCell (Nanoxis Consulting AB, www.nanoxisconsulting.com) by adding $100 \mu \mathrm{L}$ to fill the FlowCell channel (with a volume of $\sim 30 \mu \mathrm{L}$ ) using a pipette. The excess of bacterial suspension was removed from the inlet and outlet ports. The immobilized bacteria were incubated for $1 \mathrm{~h}$, at room temperature, to allow bacterial cell attachment, and the FlowCell channels were washed subsequently with $1.0 \mathrm{~mL}$ of TEAB (Triethylammonium bicarbonate) using a syringe pump, with a flow rate of $100 \mu \mathrm{L} / \mathrm{min}$. Enzymatic digestion of the bacterial proteins was performed by injecting $100 \mu \mathrm{L}$ of trypsin $(20 \mu \mathrm{g} / \mathrm{mL}$ in $200 \mathrm{mM}$ TEAB, $\mathrm{pH} \sim 8)$ into the FlowCell channels and incubating for $30 \mathrm{~min}$ at room temperature. The generated peptides were eluted by injecting $200 \mu \mathrm{l}$ TEAB $(200 \mathrm{mM}, \mathrm{pH} \sim 8)$ into the FlowCell channels at a flow rate of $100 \mu \mathrm{L} / \mathrm{min}$. The eluted peptides were collected at the outlet ports, using a pipette, and transferred into Axygen tubes $(2 \mathrm{ml})$. The peptide solutions were incubated at room temperature overnight, to allow for complete digestion, and subsequently frozen at $-20{ }^{\circ} \mathrm{C}$. As described above, each of the three biological replicates at both conditions were analyzed using triplicate samples of $\mathrm{pH} 7$ and $\mathrm{pH} 9$ (technical replicates) in order to allow for technical variation and to give statistical support for the $t$-test analysis.

The digested samples were concentrated to $30 \mu \mathrm{l}$ and $70 \mu \mathrm{l}$ of $0.5 \mathrm{M}$ TEAB (Triethylammonium Bicarbonate) was added to each tube prior to labeling with the $\mathrm{TMT}^{\circ}$ according to the manufacturer's instructions (Thermo Scientific). In a set, each sample was labeled with a unique tag from a TMT 6plex isobaric mass tag labeling kit. After TMT labeling, the samples in a set were pooled resulting in three independent sets in total to cover all samples.

\section{LC-MS/MS Analysis on LTQ-Orbitrap Velos and Q-Exactive}

Each set was divided in two equal volumes into two separate samples (sample 1 and sample 2) that were either subjected to LCMS-analysis directly (1D-LC) or further purified and fractionated by Strong Cation Exchange Chromatography (SCX) followed by LCMS-analysis (2D-LC). Sample 1, analyzed according to the 1D-LC approach, was desalted using PepClean C18 spin columns (Thermo Fisher Scientific) according to the manufacturer's guidelines prior to LCMS-analysis. The second sample (sample 2) was fractionated using SCX spin columns (Thermo Fisher Scientific) into 8 fractions according to the manufacturer's guidelines followed by a desalting step of each fraction.

Samples were reconstituted with $15 \mu \mathrm{l}$ of $0.1 \%$ formic acid (Sigma Aldrich) in 3\% acetonitrile (Sigma Aldrich) and analyzed on either an LTQ-Orbitrap Velos or Qexactive (Thermo Fisher Scientific, Inc., Waltham, MA, USA) mass spectrometer interfaced to an Easy-nLC II (Thermo Fisher Scientific). Peptides $(2 \mu \mathrm{L}$ injection volume) were separated using an in-house constructed analytical column $(200 \times 0.075 \mathrm{~mm}$ I.D. $)$ packed with $3 \mu \mathrm{m}$ Reprosil-Pur C18-AQ particles (Dr. Maisch, Germany). Solvent A was $0.2 \%$ formic acid in water and solvent B was $0.2 \%$ formic acid in acetonitrile. The following gradient was run at $200 \mathrm{~nL} / \mathrm{min} ; 5-30 \%$ B over $75 \mathrm{~min}$, $30-80 \%$ B over 5 min, with a final hold at $80 \%$ B for $10 \mathrm{~min}$. Ions were injected into the mass spectrometer under a spray voltage of $1.6 \mathrm{kV}$ in positive ion mode. The MS scans was performed at 30000 and 70000 resolution (at $\mathrm{m} / \mathrm{z} 200$ ) with a mass range of $\mathrm{m} / z$ 400-1800 for the Velos and Q-exactive, respectively. MS/MS analysis was performed in a data-dependent mode, with the top ten most abundant doubly or multiply charged 
precursor ions in each MS scan selected for fragmentation (MS/MS) by stepped high energy collision dissociation (stepped HCD) of NCE-value of 25, 35 and 45. For MS/MS scans the resolution was 7500 and 35,000 (at $m / z 200$ ) for the Velos and Q-exactive with a mass range of $\mathrm{m} / z$ 100-2000. The isolation window was set to $1.2 \mathrm{Da}$, intensity threshold of $1.1 \mathrm{e} 4$ and a dynamic exclusion of $30 \mathrm{~s}$, enabling most of the co-eluting precursors to be selected for MS/MS. Samples analyzed according to the 1D-LC approach were re-analyzed twice with exclusion lists generated after database searching of previous LCMS runs (see below).

\section{Database search for protein TMT quantification}

For relative quantification and identification the MS raw data files for each TMT set were merged in the search using Proteome Discoverer version 1.4 (Thermo Fisher Scientific). For the 1D-LC and 2D-LC approaches, the triplicate injections and the SCX fraction were combined, respectively. A database search for each set was performed with the Mascot search engine (Matrix Science LTD) using species-specific databases downloaded from Uniprot. The data was searched with MS peptide tolerance of $10 \mathrm{ppm}$ for Orbitrap Velos and $5 \mathrm{ppm}$ for Q-Exactive runs and MS/MS tolerance for identification of 100 millimass units $(\mathrm{mmu})$. Tryptic peptides were accepted with 1 missed cleavage and variable modifications of methionine oxidation, cysteine methylthiolation and fixed modifications of $\mathrm{N}$-terminal TMT6plex and lysine TMT6plex were selected. The detected peptide threshold in the software was set to $1 \%$ FDR (false discovery rate) for the experiments performed on the QExactive, and 5\% FDR for the experiments performed on the Velos, by searching against a reversed database. Identified proteins were grouped by sharing the same sequences to minimize redundancy. For the 1D-LC approach exclusion lists of $\mathrm{m} / \mathrm{z}$ values of the identified peptides with a two minutes retention time window was generated from the search results.

For TMT quantification, the ratios of the TMT reporter ion intensities in MS/MS spectra (m/z 126-131) from raw data sets were used to calculate fold changes between samples. Ratios were derived by Proteome Discoverer using the following criteria: fragment ion tolerance as $80 \mathrm{ppm}$ for the most confident centroid peak and missing values are replaced with minimum intensity. TMT reagent purity corrections factors are used and missing values are replaced with minimum intensity. Only peptides unique for a given protein were considered for relative quantitation, excluding those common to other isoforms or proteins of the same family. The quantification was normalized using the protein median. The results were then exported into MS Excel (Microsoft, Redmond, WA) for manual data interpretation and statistical analysis. Only peptides unique for a given protein were considered for relative quantitation, excluding those common to other isoforms or proteins of the same family.

\section{Statistical analysis}

First, proteins displaying more than $20 \%$ variation between the individual LPI channels for the three $\mathrm{pH} 7$ and the three $\mathrm{pH} 9$ channels respectively were removed. This was done by calculation the ratio of the separate TMT-labels in a group, and the average of the combined channels e.g. $126 /$ (average $126+127+128$ ). Proteins with ratios between 0.8 and 1.2 were included in the protein list. Second, a Welch's $t$-test was performed (3 technical replicates $\mathrm{pH} 7$ vs 3 technical replicates $\mathrm{pH}$ 9) and only proteins passing filter $p<0,05$ was accepted. Third, a fold change of at least 1.5 was set as a threshold to list proteins that had a relevant up or down regulation. Fourth, the proteins accepted for the biological interpretation had to be quantified in at least two of the three TMT-sets (biological replicates).

\section{Results}

Surface proteome analysis and protein annotation

To study the effect of alkaline pH on ETEC strain E2863 we used a MS-based quantitative proteomic strategy. Three biological replicates of the experiments were performed in $\mathrm{pH} 7$ and $\mathrm{pH} 9$, respectively. Tandem mass tag (TMT) labeling was used for multiplexed relative quantification of proteins in multiple samples [18]. Since we were interested in the bacterial surface proteome exposed to the environment during alkaline conditions we used the LPI methodology for surface shaving of bacteria to enrich for surface proteome [19].

The peptides generated by the LPI methodology were analyzed with two different separation strategies prior MS analysis to increase the number of detected proteins. Therefore, after eluting peptides from the LPI flow cell the combined sample was split into two equal parts (sample 1 and 2) and analyzed by either an one-dimensional (1D$\mathrm{LC}$ ) approach or a two-dimensional (2D-LC) approach including an offline strong cation exchange fractionation step prior to MS-analysis. The overall workflow is depicted in Fig. 1.

Since ETEC strain E2863 is not whole genome sequenced, a proteomic strain typing according to Karlsson et al. was performed, to identify the most similar strain to E2863 for peptide matching [19]. Strain identity typing identified $E$. coli $\mathrm{K} 011 \mathrm{FL}$ as the top ranking identity strain and it was used for peptide matching. In order to pick up ETEC specific genes, the ETEC reference strain H10407 was used. For each experiment the resulting protein matches using both K011FL and H10407 were annotated and finally all results obtained in the three independent replicates were combined (Table 1). 
Table 1 Protein matches to genomes used for matching peptides before and after $t$-test analysis $(p<0.05)$

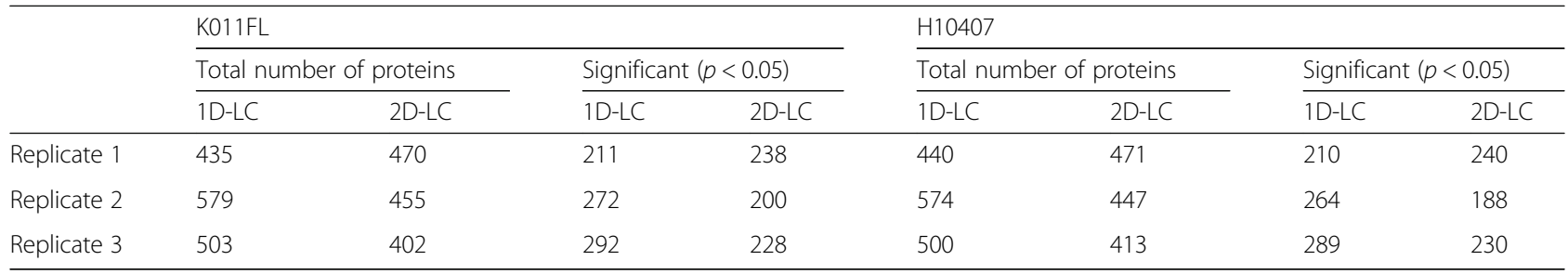

For comparison between the two $\mathrm{pH}$ conditions, foldchanges were calculated and a $p$-value $<0.05$ was considered significant (Table 1). The distribution of proteins with $P<0.05$ among the three biological replicates for both $1 \mathrm{D}-\mathrm{LC}$ and $2 \mathrm{D}-\mathrm{LC}$ is shown in Fig. 2a. In total, we included 248 proteins found in at least two of the three biological replicates for the biological interpretation. Out of the 248 proteins, 104 were found in both the 1D-LC and the $2 \mathrm{D}-\mathrm{LC}$ analysis, whereas 81 were uniquely found in the 1D-LC analysis and 63 were uniquely found in the 2D-LC analysis (Fig. 2b).

\section{Growth in alkaline conditions induce specific changes in the proteome}

The identified proteins were analyzed for up- and downregulation. In general, equal numbers of proteins and similar up- and down-regulation patterns were determined using both 1D-LC and 2D-LC. The identified proteins were grouped according to functionality and were divided into eight different categories: amino acid catabolism and transport, biosynthesis, envelope and periplasmic proteins, proton and electron transport, ribosomal, stress response, sugar catabolism and TCA cycle, and, transcription and translation. Sixty-three proteins were not grouped since most of them belong to putative or uncharacterized proteins.

We observed that identified proteins that could be grouped into the categories transcription and translation, ribosomal, proton and electron transport and periplasmic proteins were generally up-regulated under alkaline conditions compared to $\mathrm{pH} 7$. In contrast most of the proteins from sugar catabolism and TCA cycle, stress response,

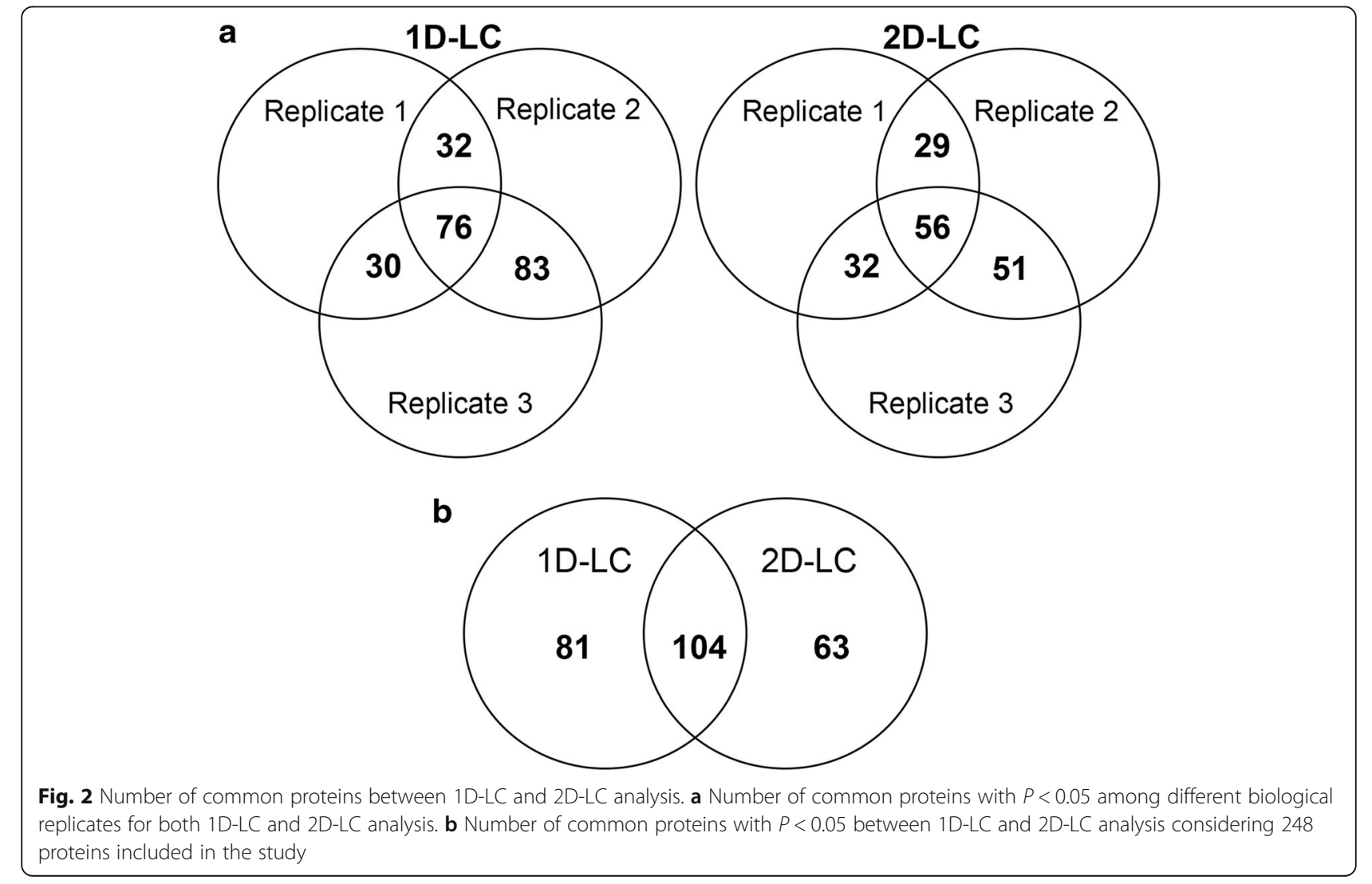


and amino acid catabolism were mainly down-regulated under alkaline conditions (Fig. 3).

Among proteins with the highest fold changes, glutamate decarboxylase $A$ and $B(\operatorname{Gad} A B)$, pyruvate oxidase (PoxB), L-asparaginase (AnsB) and nitrate reductase $(\mathrm{NarH})$ were the most down-regulated proteins at $\mathrm{pH} 9$ compared to $\mathrm{pH} 7$; whereas proteins belonging to the ATP synthase complex (AtpADFGH) were highly up-regulated. Three uncharacterized proteins were among the most down-regulated proteins at $\mathrm{pH} 9$ (i.e. a hypothetical protein: E8YA36, a putative lipoprotein: E3PFR9, and a putative stress protein: E3PC10). In addition two hypothetical proteins were among the most up-regulated (E8Y559, and E3PLV3 where the latter is predicted to be an exported protein). Although the function of these proteins is unknown, our results suggest that they are involved in alkaline $\mathrm{pH}$ responses in E. coli.

\section{Proteins involved in proton and electron transport are up-regulated at alkaline $\mathrm{pH}$}

We observed strong up-regulation of the ATP synthase operon encoding F1Fo ATP synthase, which import $\mathrm{H}^{+}$ to the cytosol during oxidative respiration [20] in contrast to down-regulation of proteins involved in the export of $\mathrm{H}^{+}$from the cytosol such as NADH ubiquinine oxireductase (NuoABCDEFGHI), nitrate reductase A $(\mathrm{NarH})$ and $\mathrm{NAD}(\mathrm{P}) \mathrm{H}$ dehydrogenase (quinone)(WrbA) (Table 2) (Fig. 4). The protein subunit of nitrate reductase $(\mathrm{NarH})$ and dimethyl sulfoxide reductase (DmsA/C) involved in the anaerobic respiration pathway were also down-regulated (Table 2). Furthermore, we observed an increase of Phage shock protein A (PspA) (Table 6), which helps maintain the proton motive force under stress conditions as well as cellular growth during alkaline and nutrient depleted environmental conditions [21]. The proteome at $\mathrm{pH} 9$ thus reflects that several membrane and periplasmic proteins are involved in retaining protons in the cytosol in order to keep a near-neutral $\mathrm{pH}$ in the cytosol at alkaline external conditions (Table 2) (Fig. 4).

\section{TCA cycle proteins are generally down-regulated at alka-} line $\mathrm{pH}$ while maltose sugar catabolism is favored

The first step in the metabolism of carbohydrates is the transport of these molecules into the cytosol. Substrates need to be transported into cells prior to their catabolic breakdown or employment for anabolic purposes. In bacteria, various carbohydrates are taken up by several mechanisms [22]. The most important transport system for carbohydrates, in particular glucose, is the phosphotransferase system (PTS). All identified enzymes of the PTS system (e.g. PtsI, PykF, Pps, PpsA and the Man system) were down-regulated (Table 3). Contrary, the proteins for maltose transport (MalEKMK) and trehalosespecific transporter (TreB) were up-regulated. Expression of genes involved in maltose or maltodextrine transport peak at exponential phase and induction of the maltose operon at alkaline $\mathrm{pH}$ has been reported in several studies [23]. It is also known that $E$. coli growing on LB utilize maltose as a preferred carbon source followed by e.g. mannose, melibiose, galactose, fucose and rhamnose [24]. In line with this the galactose/glucose import protein D-galactose-binding periplasmic protein (MglB), a

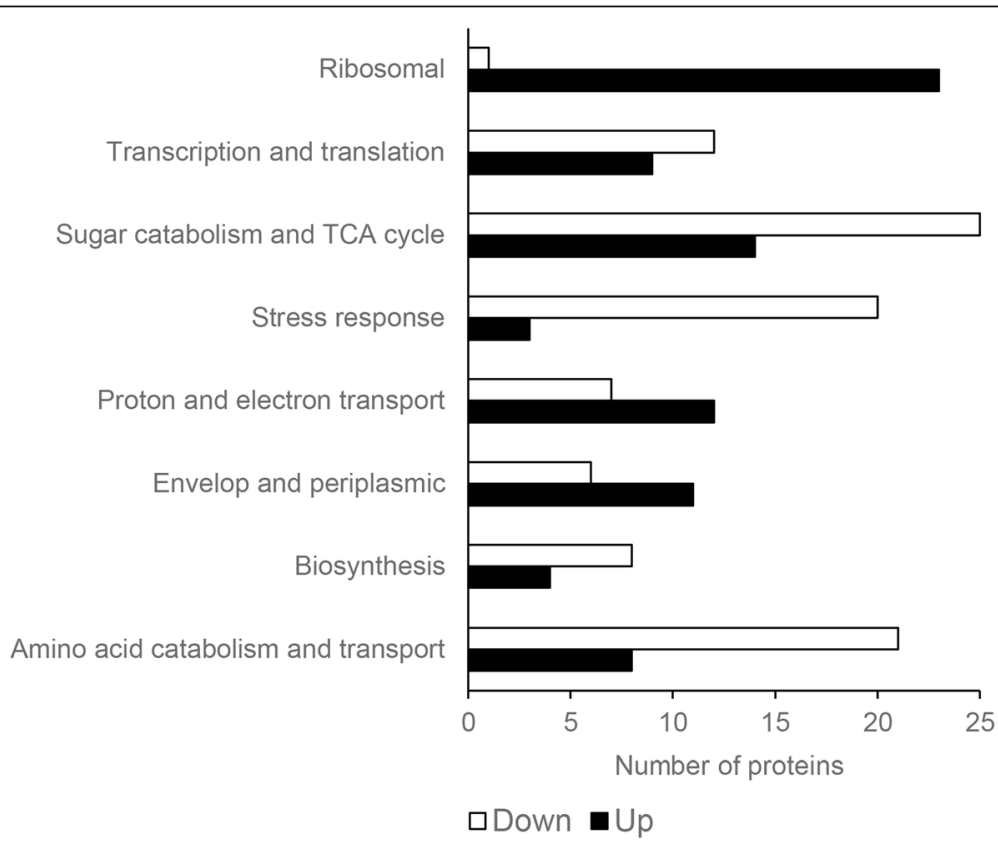

Fig. 3 Distribution of up- and down-regulated proteins among the different protein categories 
Table 2 Proteins involved in proton and electron transport

\begin{tabular}{|c|c|c|c|c|c|c|}
\hline Protein & Description & 1D-LC & 2D-LC & Regulation & Run & Matching \\
\hline AtpA & ATP synthase subunit alpha & 1.93 & 2.14 & UP & All & Both \\
\hline AtpC & ATP synthase epsilon chain & & 1.75 & UP & $2-3$ & Both \\
\hline AtpD & ATP synthase subunit beta & 1.92 & 2.14 & UP & All & Both \\
\hline AtpF & ATP synthase subunit b & 2.45 & 3.65 & UP & $2-3$ & Both \\
\hline AtpG & ATP synthase gamma chain & 2.12 & 2.82 & UP & All/1-2 & Both \\
\hline AtpH & ATP synthase subunit delta & 2.11 & & UP & $2-3$ & Both \\
\hline $\mathrm{Cmk}$ & Cytidylate kinase & & 1.60 & UP & $1-3$ & Both \\
\hline DmsA & Anaerobic dimethyl sulfoxide reductase subunit A & & 0.29 & DOWN & $2-3$ & Both \\
\hline DmsC & Anaerobic dimethyl sulfoxide reductase chain A & & 0.29 & DOWN & $2-3$ & H10407 \\
\hline $\mathrm{NarH}$ & Nitrate reductase, beta subunit & & 0.21 & DOWN & All & Both \\
\hline NirB & Nitrite reductase $(N A D(P) H)$, large subunit & 1.69 & & UP & $2-3$ & Both \\
\hline NuoF & $\mathrm{NADH}$-quinone oxidoreductase subunit $\mathrm{F}$ & 0.38 & & DOWN & $2-3$ & Both \\
\hline NuoG & $\mathrm{NADH}$-quinone oxidoreductase subunit $\mathrm{G}$ & 0.63 & 0.52 & DOWN & $2-3$ & Both \\
\hline SapE & ABC transporter ATP-binding protein & 1.67 & & UP & $2-3$ & $\mathrm{H} 10407$ \\
\hline SapF & ABC transporter, ATP-binding protein & & 1.73 & UP & $2-3$ & H10407 \\
\hline TrxA & Thioredoxin & 1.68 & & UP & $1-2$ & $\mathrm{H} 10407$ \\
\hline TrxC & Thioredoxin-like protein & 1.56 & & UP & $2-3$ & $\mathrm{H} 10407$ \\
\hline WrbA & $\mathrm{NAD}(\mathrm{P}) \mathrm{H}$ dehydrogenase & 0.23 & 0.37 & DOWN & All/2-3 & Both \\
\hline YhjA & Cytochrome $\mathrm{C}$ peroxidase & & 0.55 & DOWN & $1-3$ & Both \\
\hline
\end{tabular}

Fold changes are listed under 1D-LC and 2D-LC columns

periplasmic binding component of the galactose $\mathrm{ABC}$ transporter which is activated in response to low levels of glucose, was up-regulated, implying transport of galactose into the cell. The glucose molecule transported by MglB system is phosphorylated and converted to G6P fructose, which is then transferred and phosphorylated by the fructose PTS (EIIBCFru) system, which was up-regulated at alkaline $\mathrm{pH}$. However, other sugar transport proteins like Glycerol kinase (GlpK), involved in glycerol uptake, and UTP-glucose-1-phosphate uridylyltransferase (GalU) for galactose transport were down-regulated (Fig. 5).

The enzymes of the glycolytic pathway, the pentose phosphate pathway and TCA cycle were generally downregulated under alkaline conditions (Fig. 5). Acetate formation through pyruvate dehydrogenase (PoxB), and lactate formation through D-lactate dehydrogenase (LdhA) and phosphoenoloyruvate synthase (PpsA), which catalyzes conversions from pyruvate to PEP, does not seem to play a significant role under alkaline conditions since these proteins were down-regulated. In contrast, glucose6-phosphate 1-dehydrogenase G6PDH (Zwf), which is a key enzyme in central metabolism was up-regulated. G6PDH is involved in the distribution of carbon between glycolysis and the pentose phosphate pathway (PPP), which provides a large portion of the NADPH needed for anabolism. But G6PDH is also activated in response to oxidative stress by the soxRS regulatory system $[25,26]$.
Most identified enzymes belonging to the pentose phosphate pathway (e.g. Gnd, TktA) have previously been found in lower amounts in cells growing under alkaline conditions [27]. We observed down-regulation of both transketolase A and B (TktA, TktB) involved in the nonoxidative branch of the pentose phosphate pathway in contrast to other studies where TktA and TktB have been suggested to be regulated in opposite ways, for instance the TktB gene is induced while TktA is repressed by RpoS [28].

\section{Periplasmic and outer membrane protein transport over membranes is up-regulated at alkaline conditions}

ETEC toxin secretion has been shown to be favored by alkaline $\mathrm{pH}$ [7]. We hypothesized that alterations in the composition of proteins at the membrane and periplasmic level allows for higher secretion of LT toxin. The Sec machinery mediates translocation of LT toxin A and $B$ subunits across the inner membrane in a process that is dependent on ATP and the proton motive force. In line with this up-regulation of the Sec translocation complex (SecD/F) was observed (Table 4), In addition upregulation of YidC was also observed. YidC is an integral membrane chaperone that interacts transiently with membrane proteins during their biogenesis and stimulates their correct assembly [29]. YidC interacts with SecD and SecF to form a heterotetrameric SecDFYajCYidC accessory complex [30]. 


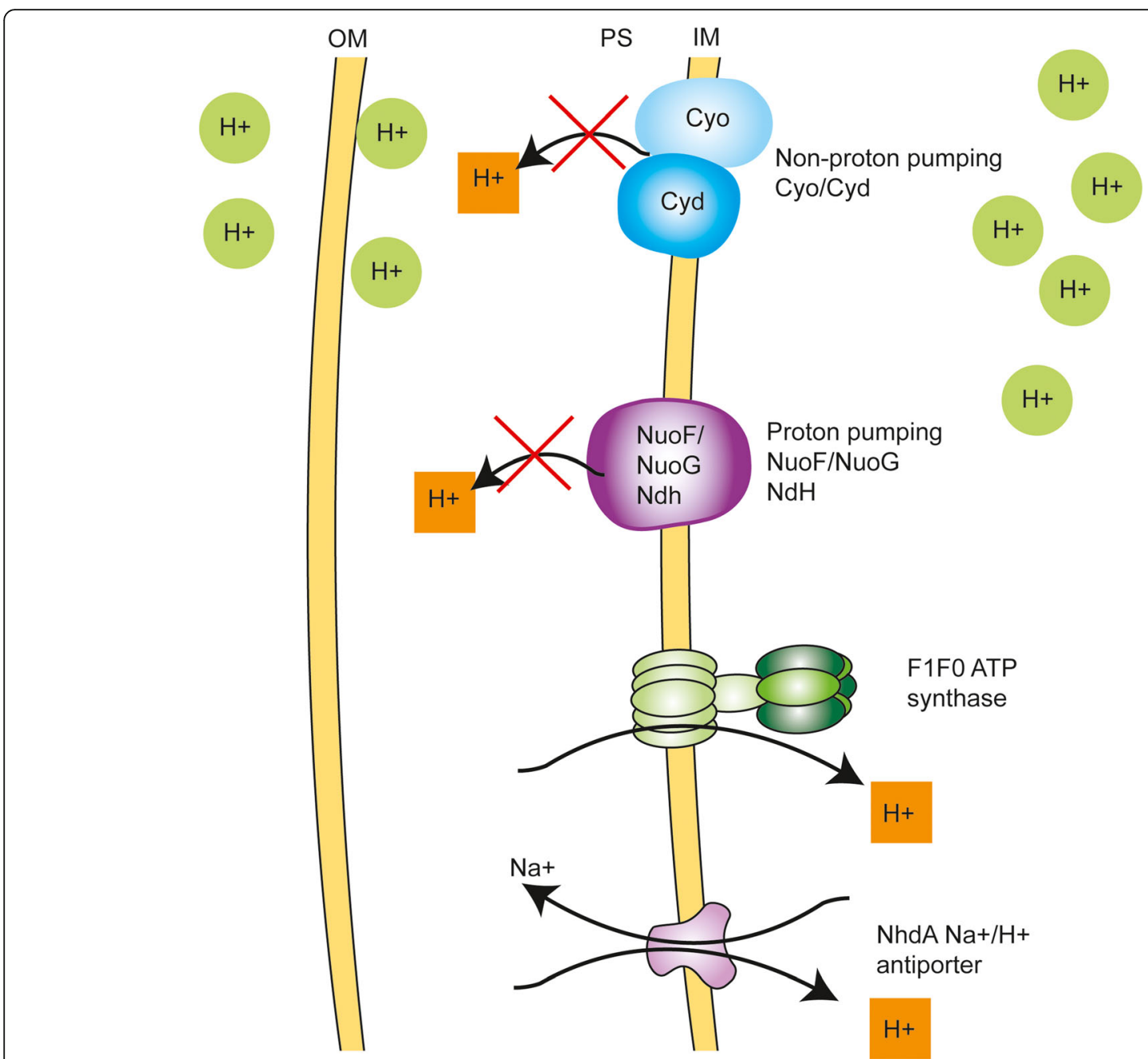

Fig. 4 Proton and electron transport system under alkaline conditions. In our system F1Fo ATP synthase, which import $\mathrm{H}^{+}$(orange) to the cytosol during oxidative respiration is up-regulated whereas proton pumping proteins NuoF, NuoG and Ndh are downregulated

In the periplasmic space the LT toxin is assembled in a $\mathrm{pH}$ - and DsbA-dependent manner and secreted through the general type II secretion pathway. Since we observed up-regulation of DsbA it is possible that increased assembly of LT holotoxin in the periplasm can explain elevated levels of secretion of LT toxin at high $\mathrm{pH}$. The Gsp components of the type II secretion pathway were however not significantly changed consistent with other findings [31].

The $\beta$-Barrel Assembly Machinery complex (BamAD) that is essential for insertion of outer membrane proteins (OMPs) in the outer membrane of gram-negative bacteria was up-regulated, in line with this the chaperone SurA that escorts outer membrane proteins to the Bam complex was induced (Table 6) as well as the outer membrane protein OmpA (Table 4). Hence, alkaline conditions might favor expression of outer membrane proteins and/or secretion in general.
The osmotic stress responses are generally down-regulated at alkaline $\mathrm{pH}$

In response to $\mathrm{pH}$ stress $E$. coli respond with different adaptive mechanisms including induction of $\mathrm{pH}$ dependent chaperones and osmoprotectants. We found that proteins involved in acidic stress response, i.e. GadAB and the acid stress induced chaperone HdeB were down-regulated as expected. In addition, trehalose-6-phosphate synthase OtsA that synthesizes the osmoprotectant trehalose under osmotic stress was down-regulated (Table 5). Additionally, two osmotically regulated permeases, ProP and ProU involved in the uptake of osmoprotectant molecules such as glycine betaine and proline were downregulated. The osmotically induced proteins OsmB/E/ Y were also down-regulated [32] (Table 5). Taken together this indicates that alkaline stress is reducing osmotic stress responses in E. coli. 
Table 3 Proteins involved in sugar catabolism and TCA cycle

\begin{tabular}{|c|c|c|c|c|c|c|}
\hline Protein & Description & 1D-LC & 2D-LC & Regulation & Run & Matching \\
\hline AceE & Pyruvate dehydrogenase E1 component & 0.62 & & DOWN & $2-3$ & Both \\
\hline AceF & Pyruvate dehydrogenase complex dihydrolipoamide acetyltransferase & 1.64 & 1.65 & UP & $2-3 / 1-3$ & Both \\
\hline AcnA & Aconitate hydratase & 0.44 & & DOWN & All & Both \\
\hline Eno & Enolase & 0.60 & & DOWN & $1-2$ & Both \\
\hline FrdA & Fumarate reductase flavoprotein subunit & 0.39 & 0.35 & DOWN & All & Both \\
\hline FrdB & Fumarate reductase iron-sulfur subunit & 0.44 & 0.36 & DOWN & All/1-2 & Both \\
\hline FruB & Bifunctional PTS system fructose-specific transporter subunit IIA/HPr protein & 1.89 & & UP & $2-3$ & Both \\
\hline FumA & Fumarate hydratase FumB & 0.26 & & DOWN & $2-3$ & Both \\
\hline GalU & UTP-glucose-1-phosphate uridylyltransferase subunit GalU & 0.65 & 0.55 & DOWN & All & Both \\
\hline GapA & Glyceraldehyde-3-phosphate dehydrogenase A & & 1.36 & UP & $1-2$ & H10407 \\
\hline GapC & Glyceraldehyde-3-phosphate dehydrogenase & 0.30 & 0.27 & DOWN & 2-3/All & Both \\
\hline GlpK & Glycerol kinase & 0.31 & 0.31 & DOWN & All & Both \\
\hline GltA & Citrate synthase & 1.62 & 1.74 & UP & 2-3/All & Both \\
\hline Gnd & 6-phosphogluconate dehydrogenase, decarboxylating & 0.43 & 0.52 & DOWN & $2-3$ & Both \\
\hline GpmA & 2,3-bisphosphoglycerate-dependent phosphoglycerate mutase & 1.70 & 1.78 & UP & $2-3$ & Both \\
\hline $\mathrm{Icd}$ & Isocitrate dehydrogenase [NADP] & & 0.87 & DOWN & All & Top \\
\hline MaeA & NAD-dependent malic enzyme & 0.62 & & DOWN & All & H10407 \\
\hline MaeB & Bifunctional malic enzyme oxidoreductase/phosphotransacetylase & 2.07 & & UP & $2-3$ & Both \\
\hline MalE & Extracellular solute-binding protein family 1 & 2.03 & 2.50 & UP & All & Both \\
\hline MalK & Maltose/maltodextrin transporter ATP-binding protein & 2.03 & & UP & $1-3$ & Both \\
\hline MalM & Maltose operon periplasmic & 1.85 & 1.88 & UP & All/2-3 & Top \\
\hline MalX & Maltose transport system, substrate-binding protein & 2.03 & 2.50 & UP & All & H10407 \\
\hline ManXYZ & PTS system mannose-specific transporter subunits $\| A B$ & 0.69 & & DOWN & $1-3$ & Top \\
\hline MglB & Methyl-galactoside $A B C$ transporter galactose-binding periplasmic protein & & 3.70 & UP & $1-2$ & Both \\
\hline PfkA & 6-phosphofructokinase & 0.48 & 0.41 & DOWN & All/2-3 & Both \\
\hline PflB & Pyruvate formate lyase I & 0.42 & 0.36 & DOWN & $1-2$ & Both \\
\hline Pgm & Phosphoglucomutase & & 0.43 & DOWN & $1-3$ & Both \\
\hline PoxB & Pyruvate dehydrogenase & 0.28 & 0.08 & DOWN & All/2-3 & Both \\
\hline Pps & Phosphoenolpyruvate synthase & 0.62 & 0.60 & DOWN & All/1-2 & Top \\
\hline PpsA & Phosphoenolpyruvate synthase & 0.62 & 0.60 & DOWN & All/1-2 & H10407 \\
\hline Ptsl & Phosphoenolpyruvate-protein phosphotransferase & & 0.95 & DOWN & All & Both \\
\hline PykF & Pyruvate kinase & 0.54 & 0.41 & DOWN & All & Both \\
\hline SucA & Succinyl-CoA ligase [ADP-forming] subunit alpha & 0.52 & 0.45 & DOWN & All & H10407 \\
\hline SucC & Succinyl-CoA ligase [ADP-forming] subunit beta & 0.39 & & DOWN & $2-3$ & Both \\
\hline SucD & Succinyl-CoA ligase [ADP-forming] subunit alpha & 0.56 & 0.45 & DOWN & $1-2 / A l l$ & Top \\
\hline TktA & Transketolase & 0.52 & 0.47 & DOWN & All & Both \\
\hline TktB & Transketolase & 0.52 & 0.49 & DOWN & $2-3 / 1-2$ & Both \\
\hline TreB & PTS system trehalose(Maltose)-specific transporter subunits IIBC & 2.01 & 2.07 & UP & 2-3/All & Both \\
\hline Zwf & Glucose-6-phosphate 1-dehydrogenase & 2.23 & 2.54 & UP & $2-3$ & Both \\
\hline
\end{tabular}

Fold changes are listed under 1D-LC and 2D-LC columns

The heat shock response is one of the main global regulatory networks in all organisms and involves an increased cellular level of chaperones and proteases to enable correct protein folding and balanced growth under different stress conditions [33]. The heat shock response in E. coli is mediated by $\sigma 32$ [33]. Among the heat shock proteins that passed our criteria for changed expression we found that DnaK and ClpAB 


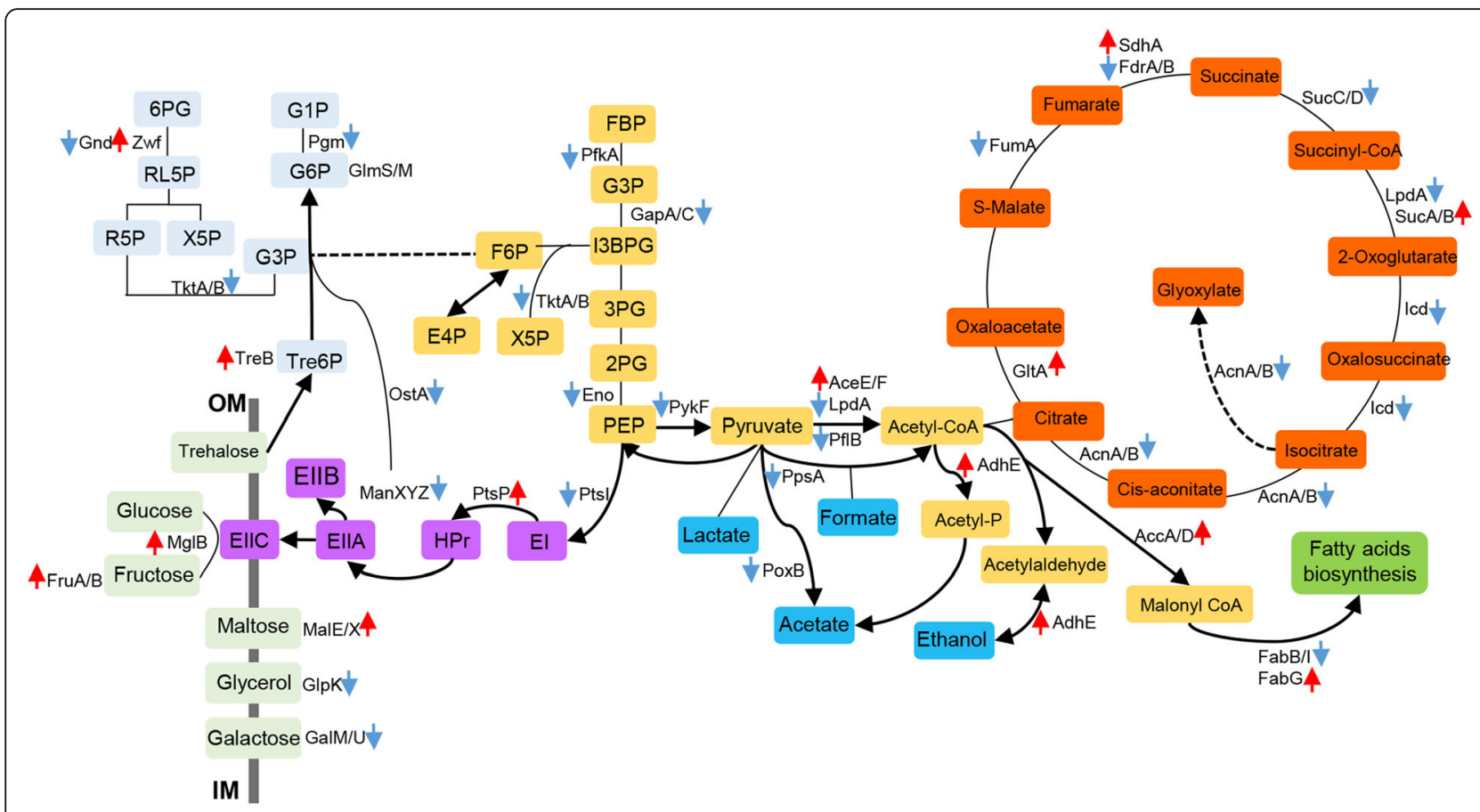

Fig. 5 Schematic representation of the sugar catabolism system and TCA cycle under alkaline conditions

were repressed in response to alkaline stress. These results were in contrast with earlier findings that have indicated that DnaK is induced by alkaline conditions [34] but supported by findings of Maurer et al. [35]. We found that the heat shock protein GroEL was upregulated consistent with other reports [34]. We also found that the cold shock protein E CspE was upregulated. Among proteases, DegP was up-regulated and PepD was down-regulated. DegP degrades abnormal proteins in the periplasm, including mutant proteins, oxidatively damaged proteins and aggregated proteins [36] (Table 5).

Table 4 Envelop and periplasmic proteins

\begin{tabular}{|c|c|c|c|c|c|c|}
\hline Protein & Description & 1D-LC & $2 \mathrm{D}-\mathrm{LC}$ & Regulation & Run & Matching \\
\hline AcrA & Acriflavin resistance protein $\mathrm{A}$ & 2.54 & 2.78 & UP & $2-3 / 1-3$ & Both \\
\hline BamA & Outer membrane protein assembly factor BamA & & 1.50 & UP & $1-2$ & H10407 \\
\hline BamD & Outer membrane protein assembly factor BamD & & 1.55 & UP & All & Both \\
\hline CopA & Copper exporting ATPase & & 0.44 & DOWN & $2-3$ & Both \\
\hline DsbA & Thiol:disulfide interchange protein & 2.33 & 2.00 & UP & All/2-3 & Both \\
\hline HchA & Molecular chaperone Hsp31 and glyoxalase 3 & & 0.41 & DOWN & All & Both \\
\hline OmpA & Outer membrane protein $\mathrm{A}$ & & 1.60 & UP & $2-3$ & Top \\
\hline $\operatorname{Sec} B$ & Protein-export protein SecB & 0.29 & 0.29 & DOWN & All & Both \\
\hline $\operatorname{Sec} D$ & Protein translocase subunit SecD & 1.67 & & UP & All & Both \\
\hline SecF & Protein-export membrane protein SecF & & 1.77 & UP & $2-3$ & Both \\
\hline SlyB & Outer membrane lipoprotein SlyB & 1.54 & & UP & $1-2$ & Top \\
\hline TraT & Putative complement resistance protein TraT & 0.52 & & DOWN & $1-3$ & H10407 \\
\hline YbiT & ABC transporter ATP-binding protein & 1.67 & & UP & $2-3$ & Top \\
\hline YbjP & Putative lipoprotein & 0.52 & 0.46 & DOWN & $1-2 / 2-3$ & Top \\
\hline YidC & Membrane protein insertase YidC & 2.04 & 1.86 & UP & All & Both \\
\hline YjjK & $A B C$ transporter related protein & & 1.34 & UP & All & Top \\
\hline YtfQ & Periplasmic binding protein/Lacl transcriptional regulator & 0.38 & & DOWN & $2-3$ & Top \\
\hline
\end{tabular}

Fold changes are listed under 1D-LC and 2D-LC columns 
Table 5 Proteins involved in biosynthesis and stress response

\begin{tabular}{|c|c|c|c|c|c|c|}
\hline Protein & Description & $1 \mathrm{DLC}$ & $2 \mathrm{DLC}$ & Regulation & Run & Matching \\
\hline$A c c B$ & Acetyl-CoA carboxylase biotin carboxyl carrier & 1.83 & & UP & $1-3$ & Both \\
\hline$A c c D$ & Acetyl-coenzyme A carboxylase carboxyl transferase & 1.53 & 1.93 & UP & $2-3$ & Both \\
\hline ClpA & ATP-dependent Clp protease ATP-binding subunit & 0.66 & & DOWN & $1-2$ & $\mathrm{H} 10407$ \\
\hline ClpB & ATP-dependent chaperone ClpB & 0.50 & 0.48 & DOWN & $1-2 / A l l$ & Top \\
\hline CspE & Cold shock protein CspE & 1.72 & 1.74 & UP & $2-3 / 1-2$ & Both \\
\hline DdlA & D-alanine-D-alanine ligase & & 0.44 & DOWN & $1-3$ & Both \\
\hline Dnak & Chaperone protein DnaK & 0.63 & 0.59 & DOWN & All & Both \\
\hline ErfK & ErfK/YbiS/YcfS/YnhG family protein & 0.64 & & DOWN & $2-3$ & Top \\
\hline FabB & 3-oxoacyl-(Acyl carrier protein) synthase I & 0.59 & 0.49 & DOWN & All & Both \\
\hline FabF & 3-oxoacyl-[acyl-carrier-protein] synthase 2 & 0.61 & & DOWN & $2-3$ & Both \\
\hline GadA & Glutamate decarboxylase & 0.18 & 0.06 & DOWN & $1-2 / 2-3$ & Both \\
\hline GadB & Glutamate decarboxylase beta subunit & 0.08 & 0.04 & DOWN & All/2-3 & $\mathrm{H} 10407$ \\
\hline GlmM & Phosphoglucosamine mutase & 0.64 & & DOWN & $1-3$ & Top \\
\hline GImS & Glutamine-fructose-6-phosphate aminotransferase & & 0.61 & DOWN & All & Both \\
\hline Glns & Glutamine-tRNA ligase & 0.52 & & DOWN & $2-3$ & Both \\
\hline Gmk & Guanylate kinase & 0.42 & & DOWN & $2-3$ & Both \\
\hline GrcA & Autonomous glycyl radical cofactor & 0.41 & 0.32 & DOWN & All & H10407 \\
\hline GroEL & 60 kDa chaperonin & 2.62 & 2.79 & UP & $2-3$ & Both \\
\hline GshB & Glutathione synthetase & & 0.46 & DOWN & $2-3$ & Both \\
\hline HdeB & Acid stress chaperone HdeB & 0.22 & & DOWN & $2-3$ & Both \\
\hline HdhA & 7-alpha-hydroxysteroid dehydrogenase & 0.27 & & DOWN & $2-3$ & Both \\
\hline $\mathrm{HtpB}$ & Chaperone protein $\mathrm{HtpG}$ & & 0.74 & DOWN & $1-3$ & Top \\
\hline KatG & Catalase-peroxidase & & 1.90 & UP & $2-3$ & Both \\
\hline LpxA & Acyl-UDP-N-acetylglucosamine O-acyltransferase & 1.51 & & UP & $2-3$ & Top \\
\hline MsyB & SecY/secA suppressor protein & 0.58 & & DOWN & $2-3$ & Both \\
\hline NapA & Periplasmic nitrate reductase & 0.46 & & DOWN & $1-3$ & Both \\
\hline OsmB & Osmotically inducible lipoprotein E & 0.46 & 0.36 & DOWN & $1-3 / A l l$ & H10407 \\
\hline OsmE & DNA-binding transcriptional activator OsmE & 0.46 & 0.36 & DOWN & $1-3 /$ All & Top \\
\hline OsmY & Osmotically-inducible protein $Y$ & 0.28 & 0.26 & DOWN & All & Both \\
\hline OtsA & Alpha,alpha-trehalose-phosphate synthase & 0.67 & & DOWN & $1-2$ & Both \\
\hline Prs & Ribose-phosphate pyrophosphokinase & & 1.60 & UP & $2-3$ & Top \\
\hline PurA & Adenylosuccinate synthetase & 0.64 & 0.62 & DOWN & All/2-3 & Both \\
\hline Skp & Chaperone protein skp & 0.59 & & DOWN & $2-3$ & Both \\
\hline SspA & Glutathione S-transferase domain protein & 0.66 & 0.55 & DOWN & $1-3$ & Both \\
\hline YbaY & Glycoprotein/polysaccharide metabolism & 0.21 & & DOWN & $2-3$ & Top \\
\hline
\end{tabular}

Fold changes are listed under 1D-LC and 2D-LC columns

Translation and transcription is induced at alkaline $\mathrm{pH}$ Proteins involved in translation were mainly up-regulated such as ribosomal proteins, ribosomal associated proteins, elongation factors, peptide chain release factor PrfC, RNA degradation protein Pnp and the initiation factor InfB. InfB was been previously reported to be produced in response to stress conditions since accumulation of $\operatorname{InfB}$ seems to be correlated with a stop of protein synthesis in E. coli [37]. These results suggest an increased production of proteins in response to alkaline stress or alternatively an increased re-localization of ribosomes to the inner membrane.

Under alkaline stress, RNA polymerase proteins RpoA/B/C were up-regulated suggesting that transcription was favored. Among transcriptional factors, the global regulators ArcA, IhfA/B and Crp were downregulated (Table 6). Proteins involved in ATP generation in the absence of oxygen or other electron acceptors, 
Table 6 Proteins involved in transcription and translation mechanisms

\begin{tabular}{|c|c|c|c|c|c|c|}
\hline Protein & Description & 1D-LC & 2D-LC & Regulation & Run & Matching \\
\hline$\overline{A r c A}$ & Aerobic respiration control protein & 0.63 & & DOWN & All & Both \\
\hline Crp & Cyclic AMP receptor protein & & 0.65 & DOWN & $1-3$ & Both \\
\hline DegP & Protease Do & 1.82 & 2.16 & UP & All & Top \\
\hline Dps & DNA protection during starvation protein & 0.39 & 0.28 & DOWN & $2-3$ & Both \\
\hline GreA & Transcription elongation factor GreA & 1.89 & & UP & $2-3$ & Both \\
\hline $\mathrm{HflC}$ & Modulator of FtsH protease $\mathrm{HflC}$ & 2.01 & 1.89 & UP & 1-3/All & H10407 \\
\hline HfIK & FtsH protease regulator $\mathrm{HflK}$ & 1.60 & 2.27 & UP & 1-3/All & Both \\
\hline $\mathrm{HflX}$ & GTPase HflX & 1.60 & 2.27 & UP & $1-3 /$ All & H10407 \\
\hline IhfA & Integration host factor subunit alpha & 0.62 & 0.63 & DOWN & All/2-3 & Both \\
\hline LepA & Elongation factor 4 & & 1.65 & UP & All & Both \\
\hline LepB & Signal peptidase I & & 1.68 & UP & $1-2$ & Both \\
\hline $\mathrm{NrdA}$ & Ribonucleoside-diphosphate reductase & 0.53 & 0.57 & DOWN & 2-3/All & Both \\
\hline $\mathrm{NrdD}$ & Anaerobic ribonucleoside triphosphate reductase & 0.56 & & DOWN & $2-3$ & Top \\
\hline NusA & NusA antitermination factor & 0.63 & & DOWN & $2-3$ & Top \\
\hline PepD & Aminoacyl-histidine dipeptidase & 0.64 & & DOWN & $1-2$ & Top \\
\hline Pnp & Polyribonucleotide nucleotidyltransferase & 1.58 & 1.78 & UP & All & Both \\
\hline PolA & DNA polymerase I & & 0.65 & DOWN & $1-2$ & Top \\
\hline PrfC & Peptide chain release factor 3 & & 1.54 & UP & $1-2$ & Both \\
\hline PspA & Phage shock protein $\mathrm{A}$ & 1.69 & 2.27 & UP & All/2-3 & Both \\
\hline RaiA & Sigma 54 modulation protein/ribosomal & 1.55 & & UP & $2-3$ & Top \\
\hline Rho & Transcription elongation protein & 0.63 & & DOWN & $2-3$ & H10407 \\
\hline RlpK & $50 S$ ribosomal protein L11 & 1.54 & 1.78 & UP & $1-3$ & Both \\
\hline Rph & Ribonuclease PH & 1.67 & 2.04 & UP & All/2-3 & Both \\
\hline RplC & $50 S$ ribosomal protein L3 & 1.54 & 1.64 & UP & All/1-3 & Both \\
\hline RplD & $50 S$ ribosomal protein $L 4$ & 1.31 & & UP & $1-3$ & Top \\
\hline RplF & $50 S$ ribosomal protein L6 & 1.28 & & UP & $1-3$ & Top \\
\hline Rpll & $50 S$ ribosomal protein $L 9$ & 1.70 & 1.87 & UP & All/2-3 & Both \\
\hline RplK & 50 S ribosomal protein L11 & 1.53 & 1.78 & UP & $1-3$ & Top \\
\hline RplO & $50 S$ ribosomal protein L15 & & 1.87 & UP & $1-3$ & Both \\
\hline RplT & 50 S ribosomal protein L20 & 1.73 & 1.86 & UP & All & Both \\
\hline RpmE & $50 S$ ribosomal protein L31 & 1.98 & & UP & All & Both \\
\hline RpmG & 50 S ribosomal protein $\mathrm{L} 33$ & & 1.95 & UP & $1-3$ & Both \\
\hline RpoA & DNA-directed RNA polymerase subunit alpha & & 1.66 & UP & $2-3$ & Both \\
\hline RpoB & DNA-directed RNA polymerase subunit beta & 1.84 & 1.66 & UP & 2-3/All & Both \\
\hline RpoC & DNA-directed RNA polymerase subunit beta' & 1.92 & 1.71 & UP & 2-3/All & Both \\
\hline RpsA & $30 \mathrm{~S}$ ribosomal protein S1 & & 1.40 & UP & $1-3$ & H10407 \\
\hline RpsH & $30 \mathrm{~S}$ ribosomal protein $\mathrm{S} 8$ & & 1.69 & UP & $2-3$ & Both \\
\hline Rps」 & $30 \mathrm{~S}$ ribosomal protein $\mathrm{S} 10$ & 1.52 & & UP & $1-3$ & Top \\
\hline RpsK & $30 \mathrm{~S}$ ribosomal protein S11 & & 2.53 & UP & All & Both \\
\hline $\mathrm{RpsO}$ & $30 \mathrm{~S}$ ribosomal protein S15 & 1.52 & 1.53 & UP & $2-3$ & H10407 \\
\hline Sra & Stationary phase induced ribosome associated protein & 0.55 & & DOWN & $2-3$ & H10407 \\
\hline Ssb & Single-stranded DNA-binding protein & 1.80 & 1.95 & UP & $1-2 / 2-3$ & Both \\
\hline SurA & Chaperone SurA & & 1.82 & UP & $1-3$ & Both \\
\hline UspF & Universal stress protein $\mathrm{F}$ & 0.43 & 0.42 & DOWN & 2-3/All & Top \\
\hline YgfZ & tRNA-modifying protein YgfZ & & 0.52 & DOWN & $1-3$ & Both \\
\hline
\end{tabular}

Fold changes are listed under 1D-LC and 2D-LC columns 
which are positively regulated by the transcriptional regulator ArcA, i.e. Ppc involved in succinate formation, and $\mathrm{PflB}$ in the pyruvate dehydrogenase complex (PDHc), were down-regulated, in contrast to up-regulation of the AceE/F complex which encode $\alpha$ and $\beta$ subunits of PDHc and is negatively regulated by ArcA. These results were consistent with the observed down-regulation of ArcA itself and indicate that alkaline $\mathrm{pH}$ repress the ArcA regulon. RpoS responsible of the expression of many genes under stress conditions was not detected in our analysis.

\section{Amino acid metabolism under alkaline conditions}

High $\mathrm{pH}$ have been reported to induce enzymes involved in generation of ammonia from amino acids including TnaA, CysK and AstD which consume tryptophan, serine, cysteine and arginine. Our results indicated a down-regulation in amino acid metabolism. TnaA, tryptophan deaminase, was down-regulated under alkaline conditions (Table 7). This result does not match with previous studies where TnaA was favored by alkaline $\mathrm{pH}$ [23]. TnaA has been reported to act as an important signaling molecule during alkaline conditions and to be regulated by RpoS under different environmental or growth conditions [38]. RpoS regulated proteins have a more important role in extended periods of stationary phase growth rather than at the onset of stationary phase [39]. It is possible that TnaA was down-regulated in our system, since the analysis of proteins was done during exponential phase. Furthermore, PutA required for the degradation of proline was up-regulated. In the presence of proline, PutA is associated with the cytoplasmic membrane and acts a bifunctional enzyme catalyzing both reactions of the proline degradation pathway: the oxidation of proline by proline dehydrogenase and subsequent oxidation to glutamate by pyrroline-5carboxylate (P5C) dehydrogenase.

\section{Discussion}

Our study highlights the effect of alkaline $\mathrm{pH}$ on the expression of proteins compared to neutral $\mathrm{pH}$ in ETEC. Since we hypothesize that infecting ETEC might encounter alkaline $\mathrm{pH}$ close to the epithelium the alkaline surface proteome might provide a better view of ETEC behavior at the site of infection and aid in identification of e.g. novel vaccine targets. However, even if our study is focused on ETEC, we have also identified general mechanisms based on the MS results that could be extrapolated to all E. coli subspecies.

The LC-MS/MS method used generated results for 3-400 proteins at each condition while E. coli is expected to express around 3000 genes of which most are translated into proteins. In this study we tried to enrich for the surface and membrane proteome, which is estimated to comprise $40 \%$ of the bacterial total proteome
[40]. Each generated sample was split into two parts and subsequently two different analysis approaches were employed, here named 1D-LC and 2D-LC. This was done in order to maximize the number of identified peptides generated by the enzymatic digestion. It has been estimated that for highly complex samples, containing 10,000-50,000 proteins in different concentration ranges, theoretically it would be necessary to be able to separate around $10 \%$ of the peptides prior to MS analysis [41]. Normally, 1D-LC approaches are not able to resolve this number of peptides, and thus multidimensional separation strategies such as $2 \mathrm{D}-\mathrm{LC}$ analysis was employed [42] to maximize the number of peptides/protein identifications.

As we used the surface shaving approach, the samples were less complex relative to a whole cell lysate, where orthogonal fractionation would be necessary. The lower complexity and the use of long separation gradients with two exclusion lists enabled the 1D separation to perform well. For a sample of low complexity, using the 2D approach will not always be beneficiary since there is a risk that some peptides, particularly of low abundance, might be lost in the first offline fractionation step. This might explain the reason for the data being complementary to each other when comparing the $1 \mathrm{D}-\mathrm{LC}$ vs $2 \mathrm{D}-\mathrm{LC}$ approach.

The two methods employed generated slightly different results. In previous shotgun proteomics studies, 1D-LC with 2D-LC approaches have been compared [41, 43]. Concerning the performance on 2D-LC approaches, the drawback of having to use offline fractionation (SCX or $\mathrm{RP})$ prior to a second online RP-LC injection of the fractions, is balanced against a higher number of peptide/protein identifications. When comparing the methodologies on the same sample, most of the identified proteins in a 1D-LC set-up are usually found also in the 2D-LC set-up [41]. In our study, however, the employed 1D-LC (RP-LC) set-up of three consecutive injections (with exclusion lists) seemed to complement the 2D-LC set-up (eight SCX fractions followed by RP-LC). The number of proteins found in both set-ups was 104 whereas 81 proteins were uniquely found in the 1D-LC set-up versus 63 proteins uniquely found in the 2D-LC set-up. This might be due to the methodology of generating the peptides, using the LPI methodology to perform surface shaving of intact bacteria. Also, it seems that hydrophobic peptides might be underrepresented when using SCX as the first dimension of analysis [41]. Further studies would be needed to explain why our approach showed that the 1D-LC and 2D-LC setups provided complimentary data instead of having a more overlapping character, this was however not the scope of this analysis.

Growth at $\mathrm{pH} 9$ poses a specific type of stress where the bacteria needs to pump $\mathrm{H}^{+}$into the cytosol to maintain a near neutral intracellular $\mathrm{pH}$ homeostasis, needed 
Table 7 Proteins involved in amino acid catabolism and transport

\begin{tabular}{|c|c|c|c|c|c|c|}
\hline Protein & Description & 1D-LC & 2D-LC & Regulation & Run & Matching \\
\hline Alas & Alanine-tRNA ligase & 0.65 & & DOWN & $2-3$ & $\mathrm{H} 10407$ \\
\hline AnsB & L-asparaginase II & 0.20 & & DOWN & $2-3$ & Top \\
\hline AroK & Shikimate kinase 1 & & 1.70 & UP & $2-3$ & Both \\
\hline AsnA & Aspartate-ammonia ligase & 0.54 & & DOWN & $2-3$ & Both \\
\hline Asns & Asparagine-tRNA ligase & 0.63 & 0.63 & DOWN & 2-3/All & Both \\
\hline AspA & Aspartate ammonia-lyase & 0.52 & 0.50 & DOWN & All/1-3 & Both \\
\hline AspS & Aspartate-tRNA ligase & 1.56 & 2.28 & UP & All & Both \\
\hline CarA & Carbamoyl-phosphate synthase small chain & & 2.53 & UP & $1-2$ & Both \\
\hline CarB & Carbamoyl-phosphate synthase large chain & 3.00 & 4.01 & UP & 2-3/All & Both \\
\hline Cysl & Sulfite reductase hemoprotein beta-component & & 0.47 & DOWN & $1-3$ & Both \\
\hline GabT & 4-aminobutyrate aminotransferase & 0.39 & & DOWN & $2-3$ & Both \\
\hline lles & Isoleucine-tRNA ligase & 0.52 & 0.62 & DOWN & 2-3/All & Both \\
\hline$\| v C$ & Ketol-acid reductoisomerase & 1.79 & & UP & $1-2$ & Top \\
\hline $\mathrm{Klb}$ & 2-amino-3-ketobutyrate coenzyme A ligase & 0.51 & & DOWN & $1-2$ & Both \\
\hline LeuS & Leucine-tRNA ligase & & 0.46 & DOWN & $2-3$ & Both \\
\hline LysS & Lysine-tRNA ligase & 0.44 & 0.40 & DOWN & All & Both \\
\hline LysU & Lysine-tRNA ligase & 0.47 & 0.38 & DOWN & All & Both \\
\hline MetG & Methionine-tRNA ligase & 0.71 & & DOWN & $1-3$ & Top \\
\hline MetK & S-adenosylmethionine synthase & 0.57 & 0.36 & DOWN & All & Both \\
\hline Phes & Phenylalanine-tRNA ligase alpha subunit & & 1.50 & UP & $1-2$ & Both \\
\hline ProP & Metabolite/H+ symporter, major facilitator superfamily & 0.40 & 0.38 & DOWN & $1-2$ & Both \\
\hline ProQ & ProP effector & & 1.87 & UP & & Both \\
\hline Prov & Glycine betaine transporter ATP-binding subunit & 0.55 & 0.63 & DOWN & $2-3$ & Both \\
\hline PutA & Delta-1-pyrroline-5-carboxylate dehydrogenase & & 1.61 & UP & $1-3$ & Both \\
\hline Sers & Serine-tRNA ligase & 0.50 & 0.45 & DOWN & All/2-3 & Both \\
\hline Tdh & L-threonine 3-dehydrogenase & & 0.57 & DOWN & $2-3$ & Both \\
\hline TnaA & Tryptophanase & 0.34 & & DOWN & $1-2$ & Both \\
\hline Tyrs & Tyrosine-tRNA ligase & 0.54 & 0.60 & DOWN & $1-2$ & Both \\
\hline Vals & Valine-tRNA ligase & & 0.66 & DOWN & $1-3$ & Top \\
\hline
\end{tabular}

Fold changes are listed under 1D-LC and 2D-LC columns

for normal function. Under $\mathrm{pH}$ stress conditions, E. coli needs to maintain the cytoplasmic $\mathrm{pH}$ between 7.2 and 7.8 in order to preserve enzymatic activity and nucleic acid and protein stability [44]. Under alkaline conditions this is executed through active influx of protons and restrained outflux of protons from the cytosol. At aerobic conditions, E. coli produce NADH and $\mathrm{FADH}_{2}$ through the TCA cycle, these reducing equivalents are oxidized in the respiratory chain, and the electrons generated from the reducing equivalents are subsequently transferred to cytochromes where $\mathrm{O}_{2}$ is converted to $\mathrm{H}_{2} \mathrm{O}$ [27]. This process is coupled to the formation of a proton motive force (PMF) over the cytoplasmic membrane, which is utilized for ATP generation from ADP and Pi through the ATP synthase complex. NADH is oxidized in the respiratory chain via a coupled NADH dehydrogenase NDH-1 encoded by Nuo and Ndh, which export $\mathrm{H}^{+}$to the periplasma while one electron is transported through the respiration system [45].

It is known that at high $\mathrm{pH}, \mathrm{NhaA}$, a sodium ion/proton antiporter uses the proton electrochemical gradient to expel sodium ions from the cytoplasm and functions primarily in the adaptation to high salinity at alkaline $\mathrm{pH}$ helps to maintain internal $\mathrm{pH}$ and to protect cells from excess sodium [46]. However, the mechanisms of regulation of internal $\mathrm{pH}$ when bacteria encounters stress conditions and how these conditions are related with virulence it is not well understood. $\mathrm{Na}^{+} / \mathrm{H}^{+}$exchange would supply intracellular bicarbonate by export of $\mathrm{H}^{+}$formed on hydration of $\mathrm{CO}_{2}$ to $\mathrm{H}^{+}$and $\mathrm{HCO}_{3-}$ [47]. In line with previous studies on $E$. coli, we confirmed that ETEC prefer ATP synthase for import of protons when alkaline stress 
occurs on cytoplasmic $\mathrm{pH}$ and prefers to minimize proton export associated with NADH-I system including Nuo, Ndh and WrbA proteins as shown in our suggested model system in Fig. 4 [35].

Our findings are largely consistent with previous studies on alkaline conditions in E coli [35, 44, 48].

We found that alkaline conditions down-regulate osmotic stress responses and these findings confirm the previously suggested link between acid stress and oxidative stress [35]. Heat-shock inducible genes such as DnaK and ClpAB were down-regulated at alkaline conditions consistent with results by Maurer et al., [35], but we also found induction of GroEL expression. We were however not able to identify alkaline tolerance proteins such us NhaA, NhaB, ChaA, MdtM, and MdfA involved in the exchange of protons for other cations [44, 49], neither the YqjA protein which has recently reported to have proton-dependent transport activity [50].

The results indicate that proteins involved in osmotic and heat-shock regulation were generally down-regulated at alkaline conditions. Secretion of LT toxin has previously been reported to be favored in the presence of salts $(\mathrm{NaCl})$ and high osmolarity (sucrose) [51]. It is possible that alkaline conditions override the need for high osmolarity but since we used buffered LBK media without $\mathrm{NaCl}$ in this study, further studies are needed to elucidate the difference between $\mathrm{Na}^{+}$and $\mathrm{K}^{+}$and its impact on ETEC virulence and secretion. In addition, since an acute infection would lead to a massive efflux of $\mathrm{Na}^{+}$and $\mathrm{Cl}^{-}$ions locally from the infected cell analyses on the effect of the infectious microenvironment on ETEC virulence would be very interesting to pursue [11].

The surface proteome analysis of ETEC was performed in order to observe changes in the outer membrane proteome that could provide insight into the surface expression at the site of infection. We were also interested to find an explanation to higher secretion level of the LT toxin under alkaline conditions [7]. We found evidence that alkaline conditions induce the secretome of ETEC since the Sec pathway was up-regulated. Export of LT subunits and several other proteins are targeted through the signal recognition particle pathway to the Sec translocon for transport peptides into the periplasm. YidC interacts with SecYEG through the SecY subunit, but also with SecD and SecF to form a heterotetrameric SecDFYajCYidC accessory complex [29, 30]. Under alkaline $\mathrm{pH}$, YidC is a relatively abundant inner membrane protein $[29,52]$. In the Sec associated form, YidC recognizes individual protein transmembrane domains in the context of the Sec translocon early during biogenesis and facilitate their folding $[29,53]$. It is hence possible that YidC is involved in specific recognition of peptides secreted during alkaline conditions but this needs to be verified. We also found that the outer membrane BAM complex as well as OmpA were upregulated under alkaline conditions.

\section{Conclusions}

Based on our results we hypothesize that ETEC adhesion to the epithelium gradually induce the outer membrane proteome and secretome including secretion of the LT toxin due to the increase in local $\mathrm{pH}$ caused by bicarbonate secreted by the deregulated CFTR channels of the infected epithelial cells. Our findings indicate that ETEC likely respond to an alkaline microenvironment close to the epithelium during infection by up-regulation of Sec dependent translocation over the inner membrane followed by increased assembly of a specific repertoire of proteins that are secreted and/or associated to the outer membrane. Additional studies are however needed to define the secretome during infection of the human gastrointestinal tract.

\section{Abbreviations \\ 1D-LCMS: One-dimentional Liquid chromatography-mass spectrometry; 2D- LCMS: Two-dimentional Liquid chromatography-mass spectrometry; CT: Cholera toxin; ETEC: Enterotoxigenic Escherichia coli; LB: Luria-Bertani media; LPI: Lipid-based protein immobilization; LT: Heat-labile toxin; MS/ MS: Tandem mass spectrometry; PMF: Proton motive force; PTS system: Phosphotransferase system; ST: Heat-stable toxin; TCA: Citric acid cycle; TMT: Tandem mass tag}

\section{Acknowledgements}

We thank Annika Thorsell at The Proteomics Core Facility at Sahlgrenska Academy, Gothenburg University for helping us with the method description in the manuscript.

\section{Funding}

This research was supported by the Swedish Research Council dnr 348-20142639, and the Swedish Foundation for Strategic Research (SSF) grant no SB12-0072 to Ås. The analysis for protein identification and quantification were performed at The Proteomics Core Facility at Sahlgrenska Academy, Gothenburg University (http://www.proteomics.cf.gu.se).

\section{Authors' contributions}

Conceived and designed the experiments: LG, Ås. Performed the experiments: LG, RK, AK, DK. Analyzed the data: LG, DK, RK, AK. Wrote the paper: LG, RK, Ås. All authors have read and approve the contents of this article.

\section{Competing interests}

Roger Karlsson and Anders Karlsson are part-time employees at Nanoxis Consulting $A B$, who holds the patent rights for the LPI-technology described in the manuscript.

\section{Consent for publication}

Not applicable.

\section{Ethics approval and consent to participate}

Not applicable.

\author{
Author details \\ ${ }^{1}$ Department of Infectious Disease, Institute of Biomedicine, Sahlgrenska \\ Academy, University of Gothenburg, SE-41346 Gothenburg, Sweden. \\ ${ }^{2}$ Nanoxis Consulting AB, SE-40016 Gothenburg, Sweden. ${ }^{3}$ Proteomics Core \\ Facility at the University of Gothenburg, SE-43050 Gothenburg, Sweden. \\ ${ }^{4}$ Department of Microbiology Tumor and Cell Biology, Karolinska Institutet, \\ Stockholm SE-17177, Sweden.
}

Received: 21 July 2016 Accepted: 13 December 2016

Published online: 07 January 2017 


\section{References}

1. Kotloff KL, Nataro JP, Blackwelder WC, Nasrin D, Farag TH, Panchalingam S, Wu Y, Sow SO, Sur D, Breiman RF, et al. Burden and aetiology of diarrhoea disease in infants and young children in developing countries (the Global Enteric Multicenter Study, GEMS): a prospective, case-control study. Lancet. 2013;382(9888):209-22.

2. Gaastra W, Svennerholm AM. Colonization factors of human enterotoxigenic Escherichia coli (ETEC). Trends Microbiol. 1996;4(11):444-52.

3. Fleckenstein JM, Hardwidge PR, Munson GP, Rasko DA, Sommerfelt $H$, Steinsland H. Molecular mechanisms of enterotoxigenic Escherichia coli infection. Microbes Infect. 2010;12(2):89-98.

4. Yu J, Webb H, Hirst TR. A homologue of the Escherichia coli DsbA protein involved in disulphide bond formation is required for enterotoxin biogenesis in Vibrio cholerae. Mol Microbiol. 1992;6(14):1949-58.

5. Mudrak B, Kuehn MJ. Heat-labile enterotoxin: beyond $\mathrm{G}(\mathrm{m} 1)$ binding. Toxins (Basel). 2010;2(6):1445-70.

6. Hirst TR, Nashar TO, Eaglestone S, Lencer WI, Webb HM, Yu J. Bacterial and host interactions during the biogenesis, toxicity and immunogenicity of Escherichia coli heat-labile enterotoxin. Biochem Soc Trans. 1994;22(2):306-9.

7. Gonzales L, Ali ZB, Nygren E, Wang Z, Karlsson S, Zhu B, Quiding-Jarbrink M, Sjoling A. Alkaline $\mathrm{pH}$ Is a signal for optimal production and secretion of the heat labile toxin, LT in enterotoxigenic Escherichia coli (ETEC). PLoS One. 2013;8(9):e74069.

8. Joffre E, von Mentzer A, Abd El Ghany M, Oezguen N, Savidge T, Dougan G, Svennerholm AM, Sjoling A. Allele variants of enterotoxigenic Escherichia coli heat-labile toxin are globally transmitted and associated with colonization factors. J Bacteriol. 2015;197(2):392-403.

9. Lasaro MA, Mathias-Santos C, Rodrigues JF, Ferreira LC. Functional and immunological characterization of a natural polymorphic variant of a heatlabile toxin (LT-I) produced by enterotoxigenic Escherichia coli (ETEC). FEMS Immunol Med Microbiol. 2009:55(1):93-9.

10. Yamanaka H, Nomura T, Fujii Y, Okamoto K. Need for TolC, an Escherichia coli outer membrane protein, in the secretion of heat-stable enterotoxin across the outer membrane. Microb Pathog. 1998;25(3):111-20.

11. Gonzales-Siles L, Sjoling A. The different ecological niches of enterotoxigenic Escherichia coli. Environ Microbiol. 2016;18(3):741-51.

12. Joffre $E$, von Mentzer A, Svennerholm AM, Sjoling A. Identification of new heatstable (STa) enterotoxin allele variants produced by human enterotoxigenic Escherichia coli (ETEC). Int J Med Microbiol. 2016;306(7):586-94.

13. Flemstrom G. Active alkalinization by amphibian gastric fundic mucosa in vitro. Am J Phys. 1977;233(1):E1-E12.

14. Flemstrom G. Cl- dependence of $\mathrm{HCO} 3$ - transport in frog gastric mucosa. Ups J Med Sci. 1980;85(3):303-9.

15. Mizumori M, Ham M, Guth PH, Engel E, Kaunitz JD, Akiba Y. Intestinal alkaline phosphatase regulates protective surface microclimate $\mathrm{pH}$ in rat duodenum. J Physiol. 2009;587(Pt 14):3651-63.

16. Aoki H, Wu H, Nakano T, Ooi Y, Daikoku E, Kohno T, Matsushita T, Sano K. Nanotransportation system for cholera toxin in Vibrio cholerae 01. Med Mol Morphol. 2009:42(1):40-6.

17. Saier Jr MH. Multiple mechanisms controlling carbon metabolism in bacteria. Biotechnol Bioeng. 1998;58(2-3):170-4.

18. Thompson A, Schafer J, Kuhn K, Kienle S, Schwarz J, Schmidt G, Neumann T, Johnstone R, Mohammed AK, Hamon C. Tandem mass tags: a novel quantification strategy for comparative analysis of complex protein mixtures by MS/MS. Anal Chem. 2003;75(8):1895-904.

19. Karlsson R, Davidson M, Svensson-Stadler L, Karlsson A, Olesen K, Carlsohn E, Moore ER. Strain-level typing and identification of bacteria using mass spectrometry-based proteomics. J Proteome Res. 2012;11(5):2710-20.

20. Kasimoglu E, Park SJ, Malek J, Tseng CP, Gunsalus RP. Transcriptional regulation of the proton-translocating ATPase (atplBEFHAGDC) operon of Escherichia coli: control by cell growth rate. J Bacteriol. 1996;178(19):5563-7.

21. Weiner $L$, Model P. Role of an Escherichia coli stress-response operon in stationary-phase survival. Proc Natl Acad Sci U S A. 1994:91(6):2191-5.

22. Gunnewijk MG, van den Bogaard PT, Veenhoff LM, Heuberger EH, de Vos WM, Kleerebezem M, Kuipers OP, Poolman B. Hierarchical control versus autoregulation of carbohydrate utilization in bacteria. J Mol Microbiol Biotechnol. 2001:3(3):401-13.

23. Stancik LM, Stancik DM, Schmidt B, Barnhart DM, Yoncheva YN, Slonczewski JL. pH-dependent expression of periplasmic proteins and amino acid catabolism in Escherichia coli. J Bacteriol. 2002;184(15):4246-58.
24. Baev MV, Baev D, Radek AJ, Campbell JW. Growth of Escherichia coli MG1655 on LB medium: monitoring utilization of sugars, alcohols, and organic acids with transcriptional microarrays. Appl Microbiol Biotechnol. 2006;71(3):310-6.

25. Zhao J, Baba T, Mori H, Shimizu K. Global metabolic response of Escherichia coli to gnd or zwf gene-knockout, based on 13C-labeling experiments and the measurement of enzyme activities. Appl Microbiol Biotechnol. 2004; 64(1):91-8.

26. Zhao J, Baba T, Mori H, Shimizu K. Effect of zwf gene knockout on the metabolism of Escherichia coli grown on glucose or acetate. Metab Eng. 2004:6(2):164-74.

27. Li Z, Nimtz M, Rinas U. The metabolic potential of Escherichia coli BL21 in defined and rich medium. Microb Cell Fact. 2014:13(1):45.

28. Weber H, Polen T, Heuveling J, Wendisch VF, Hengge R. Genome-wide analysis of the general stress response network in Escherichia coli: sigmaSdependent genes, promoters, and sigma factor selectivity. J Bacteriol. 2005; 187(5):1591-603.

29. de Gier JW, Luirink J. The ribosome and YidC. New insights into the biogenesis of Escherichia coli inner membrane proteins. EMBO Rep. 2003; 4(10):939-43.

30. Nouwen N, Driessen AJ. SecDFyajC forms a heterotetrameric complex with YidC. Mol Microbiol. 2002;44(5):1397-405

31. Dorsey FC, Fischer JF, Fleckenstein JM. Directed delivery of heat-labile enterotoxin by enterotoxigenic Escherichia coli. Cell Microbiol. 2006;8(9): $1516-27$.

32. Yim HH, Villarejo M. osmY, a new hyperosmotically inducible gene, encodes a periplasmic protein in Escherichia coli. J Bacteriol. 1992;174(11):3637-44.

33. Shimizu K. Regulation Systems of Bacteria such as Escherichia coli in Response to Nutrient Limitation and Environmental Stresses. Metabolites. 2013:4(1):1-35.

34. Taglicht D, Padan E, Oppenheim AB, Schuldiner S. An alkaline shift induces the heat shock response in Escherichia coli. J Bacteriol. 1987;169(2):885-7.

35. Maurer LM, Yohannes E, Bondurant SS, Radmacher M, Slonczewski JL. pH regulates genes for flagellar motility, catabolism, and oxidative stress in Escherichia coli K-12. J Bacteriol. 2005;187(1):304-19.

36. Skorko-Glonek J, Zurawa D, Kuczwara E, Wozniak M, Wypych Z, Lipinska B. The Escherichia coli heat shock protease HtrA participates in defense against oxidative stress. Mol Gen Genet. 1999;262(2):342-50.

37. Gutierrez-Rios RM, Freyre-Gonzalez JA, Resendis O, Collado-Vides J, Saier M, Gosset G. Identification of regulatory network topological units coordinating the genome-wide transcriptional response to glucose in Escherichia coli. BMC Microbiol. 2007;7:53.

38. Lacour S, Landini P. SigmaS-dependent gene expression at the onset of stationary phase in Escherichia coli: function of sigmaS-dependent genes and identification of their promoter sequences. J Bacteriol. 2004;186(21): 7186-95.

39. Shimizu K. Metabolic Regulation of a Bacterial Cell System with Emphasis on Escherichia coli Metabolism. ISRN Biochem. 2013;2013:645983.

40. Papanastasiou M, Orfanoudaki G, Koukaki M, Kountourakis N, Sardis MF, Aivaliotis M, Karamanou S, Economou A. The Escherichia coli peripheral inner membrane proteome. Mol Cell Proteomics. 2013;12(3):599-610.

41. Gilar M, Olivova P, Chakraborty AB, Jaworski A, Geromanos SJ, Gebler JC. Comparison of 1-D and 2-D LC MS/MS methods for proteomic analysis of human serum. Electrophoresis. 2009;30(7):1157-67.

42. Washburn MP, Wolters D, Yates 3rd JR. Large-scale analysis of the yeast proteome by multidimensional protein identification technology. Nat Biotechnol. 2001;19(3):242-7.

43. Gilar $\mathrm{M}$, Xie $\mathrm{H}$, Jaworski A. Utility of retention prediction model for investigation of peptide separation selectivity in reversed-phase liquid chromatography: impact of concentration of trifluoroacetic acid, column temperature, gradient slope and type of stationary phase. Anal Chem. 2010; 82(1):265-75.

44. Padan $\mathrm{E}$, Bibi $\mathrm{E}$, Ito $\mathrm{M}$, Krulwich TA. Alkaline $\mathrm{pH}$ homeostasis in bacteria: new insights. Biochim Biophys Acta. 2005;1717(2):67-88.

45. Friedrich T, Hellwig P. Redox-induced conformational changes within the Escherichia coli NADH ubiquinone oxidoreductase (complex I): an analysis by mutagenesis and FT-IR spectroscopy. Biochim Biophys Acta. 2010; 1797(6-7):659-63.

46. Gerchman Y, Olami Y, Rimon A, Taglicht D, Schuldiner S, Padan E. Histidine226 is part of the $\mathrm{pH}$ sensor of $\mathrm{NhaA}$, a Na+/H+ antiporter in Escherichia coli. Proc Natl Acad Sci U S A. 1993;90(4):1212-6. 
47. Praetorius J, Andreasen $D$, Jensen $B L$, Ainsworth MA, Friis UG, Johansen $T$. NHE1, NHE2, and NHE3 contribute to regulation of intracellular pH in murine duodenal epithelial cells. Am J Physiol Gastrointest Liver Physiol. 2000;278(2):G197-206.

48. Blankenhorn D, Phillips J, Slonczewski JL. Acid- and base-induced proteins during aerobic and anaerobic growth of Escherichia coli revealed by twodimensional gel electrophoresis. J Bacteriol. 1999;181(7):2209-16.

49. Lewinson O, Padan E, Bibi E. Alkalitolerance: a biological function for a multidrug transporter in pH homeostasis. Proc Natl Acad Sci U S A. 2004; 101(39):14073-8.

50. Kumar S, Doerrler WT. Escherichia coli YqjA, a Member of the Conserved DedA/Tvp38 Membrane Protein Family, Is a Putative Osmosensing Transporter Required for Growth at Alkaline pH. J Bacteriol. 2015;197(14):2292-300.

51. Trachman JD, Yasmin M. Thermo-osmoregulation of heat-labile enterotoxin expression by Escherichia coli. Curr Microbiol. 2004;49(5):353-60.

52. Chen M, Xie K, Jiang F, Yi L, Dalbey RE. YidC, a newly defined evolutionarily conserved protein, mediates membrane protein assembly in bacteria. Biol Chem. 2002;383(10):1565-72.

53. Houben EN, Urbanus ML, Van Der Laan M, Ten Hagen-Jongman CM, Driessen AJ, Brunner J, Oudega B, Luirink J. YidC and SecY mediate membrane insertion of a Type I transmembrane domain. J Biol Chem. 2002;277(39):35880-6.

\section{Submit your next manuscript to BioMed Central and we will help you at every step:}

- We accept pre-submission inquiries

- Our selector tool helps you to find the most relevant journal

- We provide round the clock customer support

- Convenient online submission

- Thorough peer review

- Inclusion in PubMed and all major indexing services

- Maximum visibility for your research

Submit your manuscript at www.biomedcentral.com/submit 